\title{
CORRECTION OF MOTION CAPTURE DATA WITH RESPECT TO KINEMATIC DATA CONSISTENCY FOR INVERSE DYNAMIC ANALYSIS
}

\author{
Wolfgang Seemann \\ Günther Stelzner \\ Christian Simonidis \\ Institut für Technische Mechanik \\ Universität Karlsruhe (TH) \\ Kaiserstr. 12 \\ 76131 Karlsruhe, Germany \\ E-mail: \{seemann, stelzner, simonidis\}@itm.uka.de
}

\begin{abstract}
Inverse dynamics analysis of human motion requires that the trajectories of the selected anatomical points are known. Therefore, standard motion capture technique by tracking marker points is generally used to obtain the trajectories. The tracking process, however, introduces high-frequency noise into the trajectories and the measured data can not be used directly to proceed in the inverse dynamic analysis. A mechanical system is consistent with kinematic data if the constraint equations of position and their time derivatives are satisfied by any parameters contained in the data set. Spurious reaction forces result from violations of the constraint equations using non consistent data. Therefore, a method is applied in this paper, whereby a new set of trajectories is generated by performing a projection of the observed positions, velocities and accelerations onto the corresponding constraint manifold to ensure the consistency of the data mentioned above. Finally, the kinematics of the system is described with the corrected data set.
\end{abstract}

\section{INTRODUCTION}

Inverse dynamic analysis is used in the studies of human motion to evaluate muscle and reaction forces between adjacent anatomical segments. There are no experimental methodologies for measuring these forces directly. Therefore, human motion studies rely on mathematical and computational models. The quality of the results is affected by the choice of the biomechanical model and by the kinematic data provided as input.
Kinematic data required for the inverse dynamic analysis of the biomechanical model can be gathered by motion capture technique. Therefore, marker points are usually attached to anatomical joints and segment extremities (Figure 1) and tracked $[1,4,11]$. The further data processing uses filter technologies to eliminate any high-frequency component arising from the digitization process in order to obtain smoother data, but this does not ensure that the kinematic data is consistent with the mechanical model adopted and the underlying kinematic constraint equations are not satisfied yet.

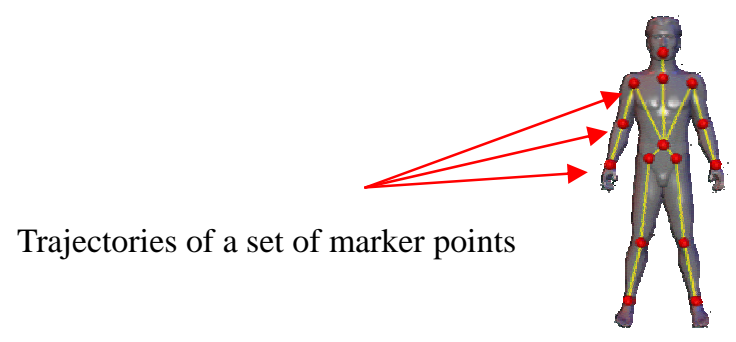

Figure 1: marker points

In the present work, a projection method is used to correct the tracked positions of the marker points to ensure non violated position constraints of the mechanical system.

Projection methods are basic components of several integration methods for differential algebraic equations. These methods all use some class of projection onto the corresponding constraint manifold. In DAE terminology the substitution of the constraints by its time derivatives is called index reduc- 
tion. Index reduction by differentiation suffers in long-time simulations from the drift-off effect. An early attempt to avoid the numerical problems of the drift-off effect in the DAE formulation goes back to the work of Baumgarte [2]. Today, it is state-of-the-art to avoid the constraint violation by projection techniques $[1,5,6,7]$. If the violation of the constraints during the time integration of the DAE formulation exceeds some error bound, then the solution is projected onto the constrained manifold. Mathematically, the projection defines a minimization problem that can be solved efficiently by Newton-Raphson iterations. The same method is applied in this paper to obtain consistent trajectories to perform an inverse dynamic analysis. A new set of trajectories is generated by performing a projection of the observed positions, velocities and accelerations onto the corresponding constraint manifold to ensure the consistency of the data. The inverse dynamic analysis is performed afterwards with the corrected and consistent input data.

The procedures mentioned above are demonstrated on a simple mechanical model and the results will be discussed with respect to the underlying principles of the techniques used. The model consists of rigid bodies which are connected by revolute joints. Inverse dynamic analysis is performed using a formalism based on natural Cartesian coordinates [9]. The position and orientation of a rigid body are defined in a global reference frame using the Cartesian coordinates of a set of basic points and unit vectors. It is convenient to use this definition for biomechanical inverse dynamic analyses since the coordinates of the digitized points can be taken directly to define the anatomical segments of the biomechanical model $[13,14]$.

\section{Rigid body definition}

Natural coordinates use points and vectors to define the position and orientation of rigid bodies [9, 12]. The rigid body constraints result of the properties of the body, i.e., distances between pairs of points on a rigid body remain constant, angles between pairs of unit vectors or between two-point segments and unit vectors remain constant. Physical relations are expressed mathematically by constraint equations, respectively. This formulation uses the scalar product to define the rigid body constraints in a unified way, as well as joint and driver constraints. Equation (1) defines the general form of the scalar product

$$
\mathbf{r}_{1}^{T} \mathbf{r}_{2}-\left|\mathbf{r}_{1}\right|\left|\mathbf{r}_{2}\right| \cos <\mathbf{r}_{1}, \mathbf{r}_{2}>=0
$$

where $\mathbf{r}_{1}$ and $\mathbf{r}_{2}$ are vectors, $\left|\mathbf{r}_{1}\right|$ and $\left|\mathbf{r}_{2}\right|$ are the respective norms and $\left\langle\mathbf{r}_{1}, \mathbf{r}_{2}\right\rangle$ is the enclosed angle between the vectors. Figure 2 shows an example of a rigid body, for which the position and orientation is defined by the position of two points $i$ and $j$ and two unit vectors $\mathbf{u}$ and $\mathbf{v}$, with related constant angles $\phi, \gamma$ and $\psi$. The position vectors of the points $i$ and $j$ in the global reference frame are defined by $\mathbf{r}_{i}$ and $\mathbf{r}_{j}$.

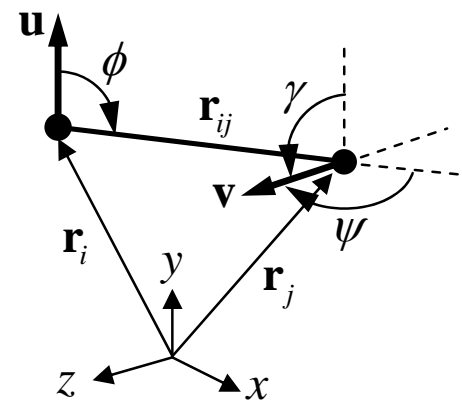

Figure 2: basic element

Thus, a vector containing the generalized coordinates to describe the position and orientation of a rigid body can be defined as:

$$
\mathbf{q}_{e}=\left[\begin{array}{llll}
\mathbf{r}_{i} & \mathbf{r}_{j} & \mathbf{u} & \mathbf{v}
\end{array}\right]^{T} .
$$

It has 12 natural coordinates. Furthermore, the degrees of freedom of the rigid element are limited to six degrees of freedom by six constraint equations:

- an equation defining the constant distance between the points $i$ and $j$ (length $l$ ),

- three equations to maintain constant angles $\phi=\gamma=\psi=$ const

- and the two conditions that $\mathbf{u}$ and $\mathbf{v}$ are unit vectors.

Thus the six constraint equations of a rigid body read:

$$
\begin{aligned}
& \mathbf{r}_{i j}{ }^{T} \mathbf{r}_{i j}-l^{2}=0 \\
& \mathbf{r}_{i j}{ }^{T} \mathbf{u}-l \cos \phi=0 \\
& \mathbf{r}_{i j}{ }^{T} \mathbf{v}-l \cos \psi=0 \\
& \mathbf{u}^{T} \mathbf{v}-\cos \gamma=0 \\
& \mathbf{u}^{T} \mathbf{u}-1=0 \\
& \mathbf{v}^{T} \mathbf{v}-1=0
\end{aligned}
$$

\section{Kinematic joint definition}

Once the constraint equations of a rigid body have been derived to guarantee the desired rigid characteristics of a body, it is necessary to formulate the constraint equations for the relative motion of the bodies. Figure 3 shows the method of modelling a spherical or a revolute joint between two rigid bodies, where $m$ and $n$ describes the name of a body respectively. The point on the joint as part of body $m$ is described by position vector $\mathbf{r}_{j}^{m}$, the point on the joint as part of body $n$ by position vector $\mathbf{r}_{i}^{n}$. 


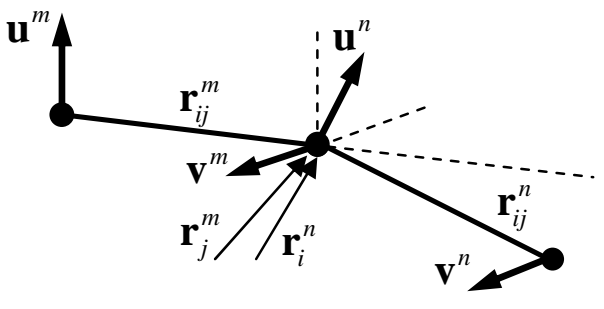

Figure 3: joint between two bodies

In case of a spherical joint both points coincide and the constraint equation results to:

$$
\mathbf{r}_{j}^{m}-\mathbf{r}_{i}^{n}=0
$$

For a revolute joint a point on a body coincides with a point on the related body. Furthermore, two angles formed by two vectors respectively have to be kept constant. Thus the constraint equations are derived to:

$$
\begin{aligned}
& \mathbf{r}_{j}^{m}-\mathbf{r}_{i}^{n}=0 \\
& \mathbf{v}^{m T} \mathbf{r}_{i j}^{n}=\text { const } \\
& \mathbf{v}^{m T} \mathbf{u}^{n}=\text { const }
\end{aligned}
$$

With the definition of the rigid body and the joints, the basic structure of the kinematic model is known. Only parameters consisting of angles and lengths need to be known to describe the complete system, yet. The rigid body constraints and the joint constraints can be rewritten into the common nonlinear position equations

$$
\mathbf{C}(\mathbf{q}, t)=\mathbf{O},
$$

where $\mathbf{q}$ is the vector of the system dependent coordinates and $t$ refers to time. Differentiation of (6) with respect to time results in

$$
\mathbf{C}_{q}(\mathbf{q}, t) \dot{\mathbf{q}}=\mathbf{C}_{t}(\mathbf{q}, t),
$$

where $\mathbf{C}_{q}$ is the Jacobian matrix and $\dot{\mathbf{q}}$ is the vector of dependent velocities. $\mathbf{C}_{t}$ is the partial derivative of the constraint equations with respect to time. For a scleronomous constraint, the corresponding entry in $\mathbf{C}_{t}$ is zero. If the position of the multi body system is known, equation (7) allows computing the dependent velocities of the multi body system. The essential difference between (6) and (7) is, that the position equations are nonlinear and the velocity equations are linear.

Differentiating the velocity equation (7) again with respect to time, the constrained equation for the accelerations is obtained as follows

$$
\mathbf{C}_{q}(\mathbf{q}, t) \ddot{\mathbf{q}}=\mathbf{Q}_{d}(\mathbf{q}, \dot{\mathbf{q}}, t),
$$

Where $\mathbf{C}_{q}$ is once more the Jacobian matrix, which was already derived before, and $\ddot{\mathbf{q}}$ is the vector of dependent accelerations. $\mathbf{Q}_{d}$ is a vector containing any quadratic terms obtained by differentiating equation (7). The acceleration problem (8) is al- ways non-homogeneous as long as the velocities are not equal to zero.

The kinematic of the multi body system is completely defined with equations (6), (7) and (8).

\section{Equation of motion}

The equations of motion for a general multi body system can be formulated by

$$
\mathbf{M} \ddot{\mathbf{q}}+\mathbf{C}_{q}^{T} \lambda=\mathbf{Q}_{e},
$$

where $\ddot{\mathbf{q}}$ is the vector of generalized acceleration, $\mathbf{M}$ is the global mass matrix of the system, $\mathbf{Q}_{e}$ is the vector of generalized external forces, $\boldsymbol{\lambda}$ is the vector of the Lagrange multipliers associated with the kinematic constraints and $\mathbf{C}_{q}$ is the Jacobian matrix.

In order to perform an inverse dynamic analysis it is assumed that the motion of the system and the external forces applied to the model are fully known. Therefore, Equation (9) is rewritten to

$$
\mathbf{C}_{q}^{T} \boldsymbol{\lambda}=\mathbf{Q}_{e}-\mathbf{M} \ddot{\mathbf{q}}
$$

Equation (10) only has a unique solution for the unknown Lagrange multipliers $\lambda$ when the number of kinematic constraints equals the number of coordinates of the system. Furthermore, the positions, velocities and accelerations have to be consistent with the kinematics of the system. This is important if inverse dynamics is applied for a given motion which is measured by motion capture for example. Such data in general are noisy and thus lead to large errors in the accelerations and therefore also to the reaction forces. However, at this point the fact can be used that the measured data should also fulfil the constrained equations (6), (7), (8) for position, velocities and acceleration. Therefore, the Cartesian coordinates of the marker points have to be modified in such a way that the kinematic constraints are fulfilled. The procedure to calculate a new set of trajectories of marker points from a given set of noisy data is accomplished by performing a kinematic analysis of the system. First, the initially non-consistent positions are used to calculate average link lengths between the marker points. These average lengths are further used to define the dimensions of the rigid bodies of the biomechanical model, so that the model has constant link lengths during the analysis.

A possibility to generate a new set of consistent input data is proposed by [14]. Therefore, the mechanical model is driven throughout the kinematic analysis, using the rotational driver constraint equations. The curves that express the history of the intersegmental angles are calculated from the input data and interpolated using cubic splines. It is then possible, to evaluate the system position at any intermediate time step. As a result, the kinematic analysis produces a new set of marker positions, which are consistent with the kinematic structure of the mechanical model.

The present work shows another possibility to generate a new set of consistent input data for the inverse dynamic analysis. Figure 4 presents an overview of the process. 


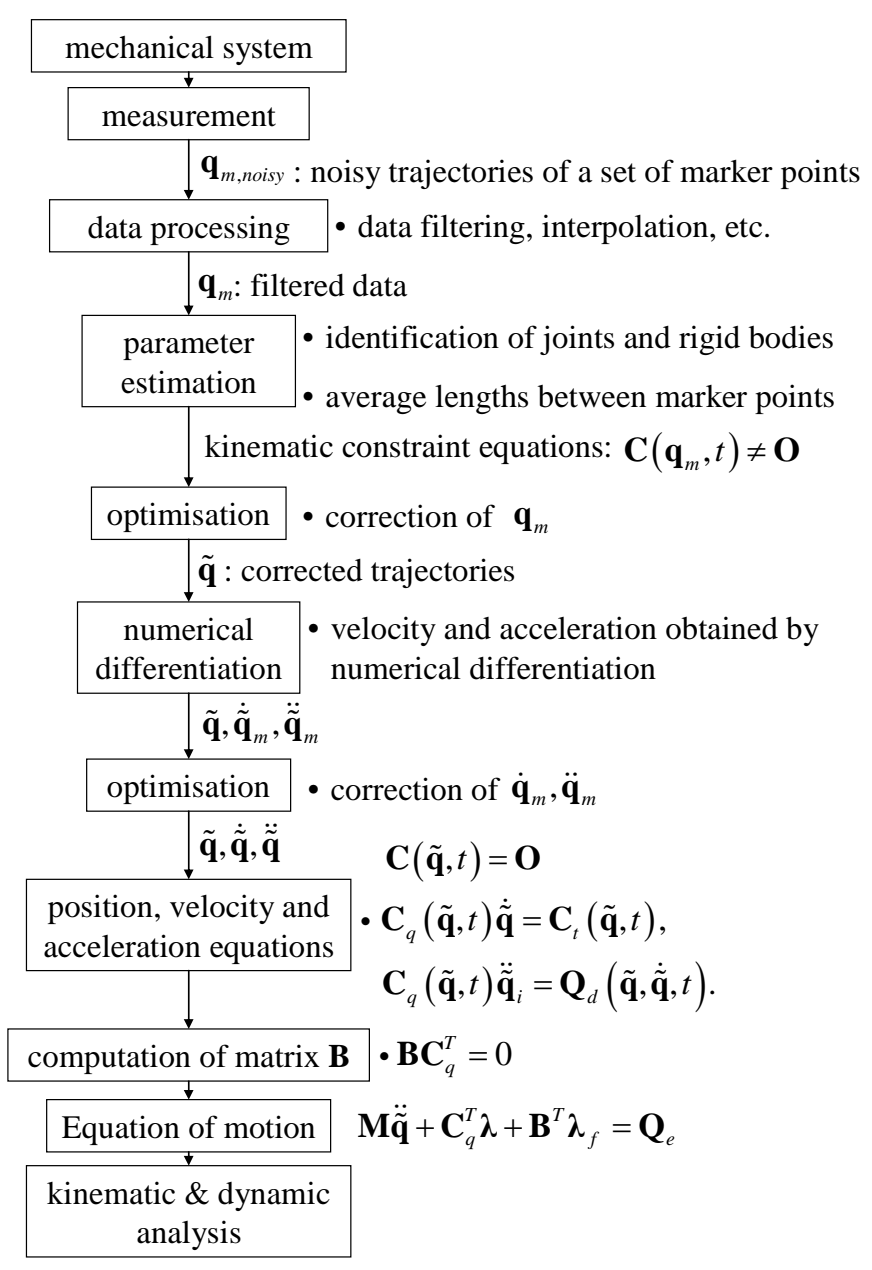

Figure 4: Overview of the data reconstruction process

The proposed approach was not tested on measured data from human motion, but rather on a much simpler system with synthetic data. This system is depicted in Figure 5 and Figure 6, which consists of four rigid bodies and four revolute joints. In order to measure the error between reconstructed and the original motion no measured data was used to test the model. Therefore, the source data was created from a reference model, for which any kind of data consisting of parameters, position, velocity, acceleration, constraint forces and driving forces was known. Then, some marker points were defined in this model and noise was applied to their trajectories to imitate the output of a motion capture procedure. The noisy data obtained was used as the only source data for further derivation of the equations of motion. Then inverse dynamics was applied assuming that mass and inertia parameters of the rigid bodies and the applied external forces are known.

\section{Mechanical System (Example)}

The previous described procedure is applied on a system with four degrees-of-freedom, which is shown in Figure 5. Four rigid bodies and four revolute joints form the rigid body system. Figure 6 visualizes the four rotation axes defined by the vectors $\mathbf{u}_{1}, \mathbf{r}_{i j}^{1}, \mathbf{v}_{2}$ and $\mathbf{u}_{4}$. The number of the resulting coordinates $\mathbf{q}$ is 48 , the number of rigid body constraints is 24 . A total of 20 joint constraints are implied by the four revolute joints. The system is driven from the initial position (see Figure 5) to the final position (see Figure 6), where the ideal model was available with any of the variables known by a numerical simulation. The simulation data is used to generate noisy input data by adding noise. Further, it can be used to evaluate the trajectories at the end. Assuming the case, that a real biomechanical model is available, most of the variables would not be known, i.e. muscle forces and joint forces.

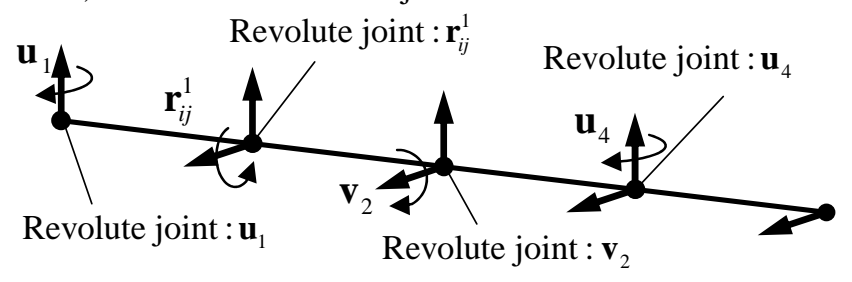

Figure 5: 4 DOF Test example

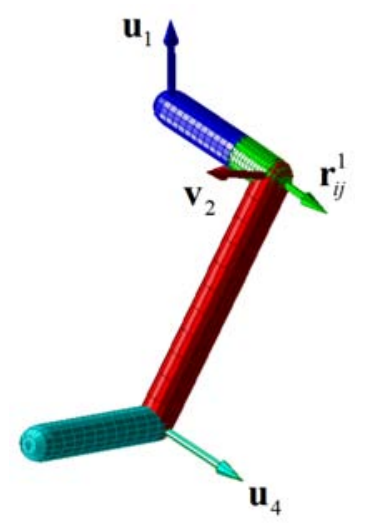

Figure 6: 4 DOF System

\section{Measurement and data processing}

The source data for a further examination is gathered from the ideal model. Figure 7 is an example for the position data processing, where the first curve describes the original data representing the reference model. Then, a random generator is used to obtain the noisy data presented by the second curve and further processing is necessary to reduce the high-frequency noise. Therefore, a filter was applied using zero-phase filtering to reduce the noise level. The third curve in Figure 7 represents the filtered data for use as the input data to calculate the kinematics. 


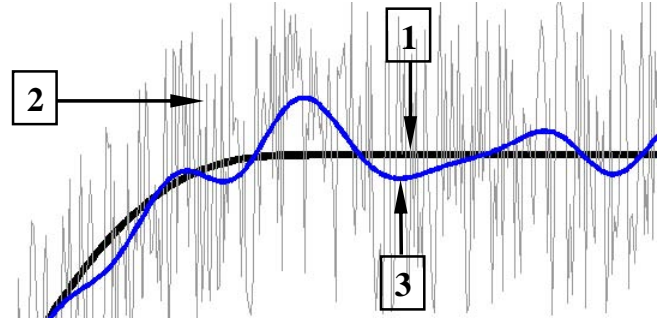

Figure 7: Example of original, noisy and filtered input data

\section{Parameter estimation}

For the kinematical structure of the system and the filtered input data, the parameters can now be calculated. But the input data obtained by the zero-phase filter is still not consistent with the kinematical structure and will lead to violations of the kinematic constraints.

These disparities are typical for motion capture techniques due to the limited resolution of the digital image as well as the positions of the markers, which are strongly affected by skin movements. Furthermore, the real anatomic joints do not describe ideal mechanical joints, as assumed by the model.

Now, the rigid bodies are identified from the available input data. Following, the trajectories are associated to the particular body and the constant average length and the constant average angles of any rigid body are determined. It is not ensured, yet, that the parameters determined are equal in time, as shown in Figure 8.
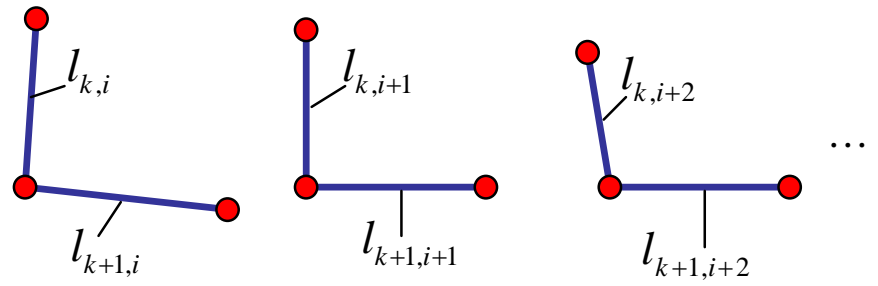

Figure 8: Distances between marker points are not constant

The process of measuring the positions of marker points during the motion results in varying distances and angles due to the inconsistent input, see Figure 8. In this figure $l_{k, i}$ refers to distance $k$ at time $t_{i}$. This means that $l_{k, i}, l_{k, i+1}, l_{k, i+2}$ corresponds to the same length at three successive time steps. Though all these lengths should be equal, this is not the case if the length is calculated using the measured and filtered data. As the real length is not known a priori it is approximately obtained by averaging the lengths over all time steps which are available. Thus, the actual input data would still cause violations of the kinematic constraints of the mechanical system. The constraint equations are then completely defined with the knowledge of the constant lengths and angles as well as the position and the types of the joints.

This part of the procedure deserves the most attention and it will be the subject for future works.

\section{Position correction}

In the following it is assumed that only holonomic and scleronomic constraints have to be fulfilled, which leads to

$$
\mathbf{C}(\mathbf{q})=\mathbf{0} \text {. }
$$

The data available from the measurement $\mathbf{q}_{m}$ do not fulfill equation (11), which results in

$$
\mathbf{C}\left(\mathbf{q}_{m}\right) \neq \mathbf{0} \text {. }
$$

It is now taken into consideration to change $\mathbf{q}_{m}$ in such a way, that $\mathbf{q}_{m}$ fulfils equation (11). Therefore, the Newton-Raphson method is applied. It is based on a linearization of the nonlinear constraint equations (11) by replacing the equations with the first two terms of its approximation by a Taylor series. Once the substitution has been made, the corrected values $\mathbf{q}$ can be obtained by

$$
\mathbf{C}\left(\mathbf{q}_{i}\right)+\mathbf{C}_{q}\left(\mathbf{q}_{i}\right)\left(\mathbf{q}-\mathbf{q}_{i}\right)=\mathbf{0} .
$$

The iterative scheme of the Newton-Rhapson method is used in terms of a least-squares approach for any time step. It has a quadratic convergence rate in the neighbourhood of the solution. Using the non-consistent positions as an initial value $\mathbf{q}_{i, r=0}=\mathbf{q}_{i, m}$, a convergent solution is obtained after two or three iterations and thus, this is a new set of corrected coordinates $\tilde{\mathbf{q}}_{i}$.

In the case of kinematical driven systems, where the number of coordinates is equal to the number of constraint equations, the recursive formula (14) for the Newton-Rhapson scheme is obtained by rewriting equation (13)

$$
\mathbf{q}_{i, r+1}=\mathbf{q}_{i, r}-\left[\mathbf{C}_{q}\left(\mathbf{q}_{i, r}\right)\right]^{-1} \mathbf{C}\left(\mathbf{q}_{i, r}\right) .
$$

In this equation, the subscript $r$ represents the iteration index and the subscript $i$ indicates the time index. The Iteration is executed until the largest error becomes smaller than a predefined error bound $\varepsilon$

$$
\max \left|\mathbf{C}\left(\mathbf{q}_{i, r+1}\right)\right|<\varepsilon \text {. }
$$

In case of a kinematical driven system, the matrix $\mathbf{C}_{q}$ is in general a square and non-singular matrix. Therefore, the related system of linear equations (14) has an exact solution.

In this work, no kinematical driver constraint equations are added to the system to obtain a square matrix $\mathbf{C}_{q}$. The present linear system in (14) includes $m$ equations with $n$ unknown variables, with $n>m$ and, thus, the Jacobian matrix $\mathbf{C}_{q}$ of the system does not have an inverse and equation (14) represents an underdetermined linear system. Its solution is not unique. A basic solution of the linear system, which has at most $m$ nozero components, can be found, but even this may not be unique.

A partial replacement for the inverse in equation (14) is provided by the Moore-Penrose pseudoinverse $[6,10]$. Therefore, the system (14) results to

$$
\mathbf{q}_{i, r+1}=\mathbf{q}_{i, r}-\left[\mathbf{C}_{q}\left(\mathbf{q}_{i, r}\right)\right]^{-1 \dagger} \mathbf{C}\left(\mathbf{q}_{i, r}\right)
$$


where $\mathbf{C}_{q}\left(\mathbf{q}_{i, r}\right)^{-1 \dagger}$ is the pseudoinverse of the Jacobian matrix. The computed solution for this system is also called the minimal norm solution because it minimizes $\left|\Delta \mathbf{q}_{i}\right|$, where

$$
\left|\Delta \mathbf{q}_{i}\right|=\left|\mathbf{q}_{i, r+1}-\mathbf{q}_{i, r}\right| \text {. }
$$

This is reasonable for the considered application to determine a correction as small as possible.

An example is introduced to show the difference between the basic and the minimal norm solution. Consider Figure 9, which shows a moving point on a circular path. It is now assumed, that the vector $\mathbf{q}$ defines the exact location of the point and the vector $\mathbf{q}_{m}$ defines the measured position. For the given system, there is one constraint equation

$$
\mathbf{C} \equiv x^{2}+y^{2}-l^{2}=0 .
$$

The Jacobian matrix $\mathbf{C}_{q}$ can be derived from the velocity equations

$$
\mathbf{C}_{q} \dot{\mathbf{q}} \equiv\left[\begin{array}{ll}
2 x & 2 y
\end{array}\right]\left[\begin{array}{c}
\dot{x} \\
\dot{y}
\end{array}\right]=0 .
$$

To calculate the new, corrected data with the recursive formula (16), the equation can be written to

$$
\left[\begin{array}{ll}
2 x_{r} & 2 y_{r}
\end{array}\right]\left[\begin{array}{l}
x_{r}-x_{r+1} \\
y_{r}-y_{r+1}
\end{array}\right]=\left[x_{r}^{2}+y_{r}^{2}-l^{2}\right] .
$$

Figure 10 shows three possibilities for a solution $\Delta \mathbf{q}$.

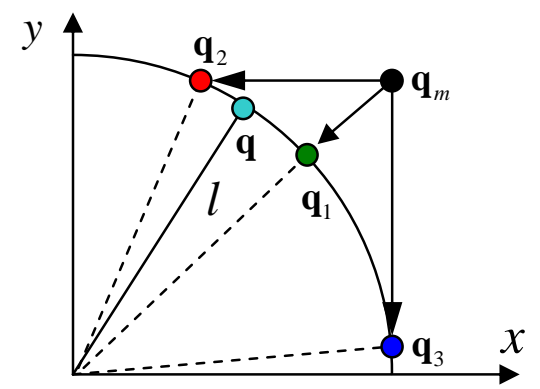

Figure 9: Moving point on a circular path

The first solution $\mathbf{q}_{1}$ is obtained by calculating the minimal norm solution of the underdetermined equation (19) with the condition (20)

$$
\left|\mathbf{q}_{1}-\mathbf{q}_{m}\right| \min \text {. }
$$

The second and the third solutions are called a base solution, which has one nonzero component. Solution $\mathbf{q}_{2}$ is given by

$$
\left[\mathbf{q}_{2}-\mathbf{q}_{m}\right]=\left[\begin{array}{c}
x_{2}-x_{m} \\
0
\end{array}\right]
$$

and solution $\mathbf{q}_{3}$ results in

$$
\left[\mathbf{q}_{3}-\mathbf{q}_{m}\right]=\left[\begin{array}{c}
0 \\
y_{3}-y_{m}
\end{array}\right] .
$$

The preferred solution is $\mathbf{q}_{1}$, which fulfills the minimal norm condition.
Any data calculated by the iteration rule (16) satisfies the position constraint equations. In the following the corrected position vectors will be named $\tilde{\mathbf{q}}_{i}$.

\section{Velocity and acceleration correction}

A mechanical system is considered to be totally consistent if its velocities and accelerations are consistent with the velocity and acceleration equations as well.

In a next step, the velocities and accelerations $\dot{\mathbf{q}}_{i, m}$ and $\ddot{\mathbf{q}}_{i, m}$ are obtained by numerical differentiation of the corrected position coordinates $\tilde{\mathbf{q}}_{i}$ with respect to time. Both are not consistent with the velocity and acceleration constraint equations. This may be expressed as follows:

$$
\begin{aligned}
& \mathbf{C}_{q}\left(\tilde{\mathbf{q}}_{i}, t\right) \dot{\mathbf{q}}_{i, m}-\mathbf{C}_{t}\left(\tilde{\mathbf{q}}_{i}, t\right) \neq \mathbf{0}, \\
& \mathbf{C}_{q}\left(\tilde{\mathbf{q}}_{i}, t\right) \ddot{\mathbf{q}}_{i, m}-\mathbf{Q}_{d}\left(\tilde{\mathbf{q}}_{i}, \dot{\mathbf{q}}_{i, m}, t\right) \neq \mathbf{0} .
\end{aligned}
$$

In this case again, a minimal norm solution can be found by minimizing the correction $\Delta \dot{\mathbf{q}}$ and $\Delta \ddot{\mathbf{q}}$ :

$$
\left|\dot{\mathbf{q}}_{i, m}-\dot{\tilde{\mathbf{q}}}_{i}\right| \min , \quad\left|\ddot{\mathbf{q}}_{i, m}-\ddot{\tilde{\mathbf{q}}}_{i}\right| \min .
$$

The goal is now to compute the corrected velocities and accelerations in such a way, that

$$
\begin{aligned}
& \mathbf{C}_{q}\left(\tilde{\mathbf{q}}_{i}, t\right) \dot{\tilde{\mathbf{q}}}_{i}=\mathbf{C}_{t}\left(\tilde{\mathbf{q}}_{i}, t\right), \\
& \mathbf{C}_{q}\left(\tilde{\mathbf{q}}_{i}, t\right) \ddot{\tilde{\mathbf{q}}}_{i}=\mathbf{Q}_{d}\left(\tilde{\mathbf{q}}_{i}, \dot{\tilde{\mathbf{q}}}_{i}, t\right) .
\end{aligned}
$$

The solution of the constrained linear least-squares problem results to the corrected generalized consistent velocities $\dot{\tilde{\mathbf{q}}}_{i}$ and accelerations $\ddot{\tilde{\mathbf{q}}}_{i}$.

\section{Inverse dynamic analysis}

The main objective of the inverse dynamic analysis is the calculation of the reaction forces and the driving forces. In order to perform the inverse dynamic analysis it is assumed that the motion (corrected positions, velocities and accelerations $\tilde{\mathbf{q}}$, $\dot{\tilde{\mathbf{q}}}, \ddot{\tilde{\mathbf{q}}}$ ), of the system and the external forces $\mathbf{Q}_{e}$ are fully known. Therefore, equation (9) is rewritten to

$$
\mathbf{M} \ddot{\tilde{\mathbf{q}}}+\mathbf{C}_{q}^{T} \boldsymbol{\lambda}+\mathbf{B}^{T} \boldsymbol{\lambda}_{f}=\mathbf{Q}_{e}
$$

where $\lambda_{f}$ are the Lagrange multipliers associated to the driving forces and $\lambda$ are the Lagrange multipliers associated to the constraint forces. Matrix $\mathbf{B}$ is calculated to fulfill the condition:

$$
\mathbf{B C}_{q}^{T}=0 \text {. }
$$

This means that the rows of $\mathbf{B}$ constitute an orthogonal basis of the nullspace of the Jacobian matrix $\mathbf{C}_{q}$. The equation of motion (26) can now be solved for the Lagrange multipliers $\left[\begin{array}{ll}\lambda & \lambda_{f}\end{array}\right]^{T}$ because the leading matrix in (28) is square and nonsingular:

$$
\left[\begin{array}{ll}
\mathbf{C}_{q}^{T} & \mathbf{B}^{T}
\end{array}\right]\left[\begin{array}{c}
\boldsymbol{\lambda} \\
\boldsymbol{\lambda}_{f}
\end{array}\right]=\mathbf{Q}_{e}-\mathbf{M} \ddot{\tilde{\mathbf{q}}} \cdot
$$


Each Lagrange multiplier is associated with a reaction force and each reaction force has a physical meaning depending on the related type of kinematic constraint. For the computation of the matrix B the Singular Value decomposition and the QR decomposition are used as follows.

\section{Method based on the Singular Value decomposition (SVD).}

The SVD $[9,12]$ decomposes a rectangular matrix $\mathbf{C}_{q}$, as indicated in the sketch in Figure 10.

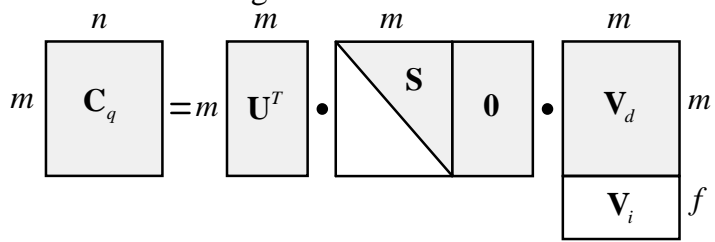

Figure 10: SV Decomposition

It can be decomposed according to equation (29) $[8,15]$,

$$
\mathbf{C}_{q}=\mathbf{U}^{T} \mathbf{S V},
$$

where matrix $\mathbf{U}$ is orthogonal and of size $m \times m$. Matrix $\mathbf{S}$ is composed of a diagonal matrix of size $m \times m$ which contains the singular values and a zero matrix given by $f=n-m$ columns ( $f$ degrees of freedom). Matrix $\mathbf{V}$ is orthogonal of size $n \times n$ and can be decomposed into two submatrices $\mathbf{V}_{d}$ and $\mathbf{V}_{i}$ of sizes $m \times n$ and $f \times n$ respectively. The most important property of the SVD is that the rows of the matrix $\mathbf{V}_{i}$ constitute an orthogonal basis of the nullspace of matrix $\mathbf{C}_{q}$. Since,

$$
\mathbf{C}_{q} \mathbf{V}_{i}^{T}=\mathbf{0}
$$

the following relationship results in:

$$
\mathbf{V}_{i}=\mathbf{B} \text {. }
$$

\section{Method based on the QR decomposition.}

This method of constructing matrix $\mathbf{B}$ is similar to the previous method. QR decomposition [9, 12] is not an iterative process, as a main advantage, and therefore, it requires fewer arithmetical operations.

The method decomposes matrix $\mathbf{C}_{q}^{T}$ as indicated in the sketch of Figure 11.

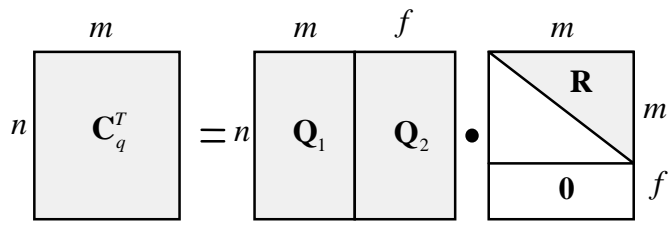

Figure 11: QR decomposition

$\mathbf{C}_{q}^{T}$ can be decomposed as shown in equation (32),

$$
\mathbf{C}_{q}^{T}=\mathbf{Q R}
$$

where $\mathbf{Q}$ is an orthogonal $n \times n$ matrix formed by an $n \times m$ matrix $\mathbf{Q}_{1}$ and an $n \times f$ matrix $\mathbf{Q}_{2}$, with:

$$
\mathbf{Q}_{1}^{T} \mathbf{Q}_{2}=0, \mathbf{Q}_{2}^{T} \mathbf{C}_{q}^{T}=0 .
$$

$\mathbf{R}$ is a rectangular $n \times m$ matrix formed by an upper triangular matrix $m \times m$ and a zero matrix of order $f \times m$. The application of this decomposition to the problem is straightforward when considering that the $f$ columns of $\mathbf{Q}$ constitute an orthogonal basis of the nullspace of matrix $\mathbf{C}_{q}$. Thus, matrix $\mathbf{B}$ can be written as

$$
\mathbf{B}=\mathbf{Q}_{2}^{T} .
$$

With known matrix $\mathbf{B}$, the solution of (28) is given by:

$$
\left[\begin{array}{c}
\lambda \\
\lambda_{f}
\end{array}\right]=\left[\begin{array}{ll}
\mathbf{C}_{q}^{T} & \mathbf{B}^{T}
\end{array}\right]^{-1}\left(\mathbf{Q}_{e}-\mathbf{M} \ddot{\mathbf{q}}\right) .
$$

The forces associated to the rigid body and joint constraints are given by $\mathbf{F}_{c}=\mathbf{C}_{q}^{T} \boldsymbol{\lambda}$. The driving forces result in $\mathbf{F}_{f}=\mathbf{C}_{q}^{T} \boldsymbol{\lambda}_{f}$.

\section{Conclusion}

Inverse dynamic analysis was carried out with and without kinematically consistent data. Significant differences could be observed. Using non consistent data, the constraint equations are violated resulting in spurious reaction forces and driver forces. In contrast, a corrected data set was calculated and the resulting consistent positions, velocities and accelerations match the reference data very well (Figure 12).
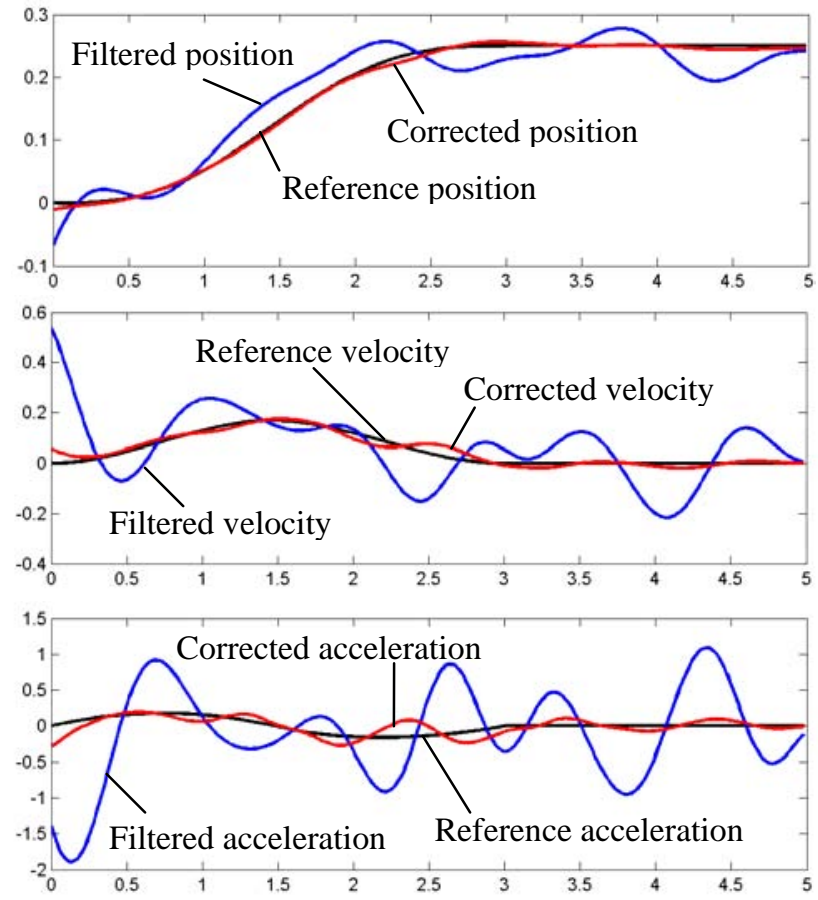

Figure 12: Comparing the reference, filtered and corrected positions, velocities and accelerations

Furthermore, reaction forces resulting from consistent kinematic data match the original data better than the forces obtained with filtered data without kinematical correction (see Figure 13). 


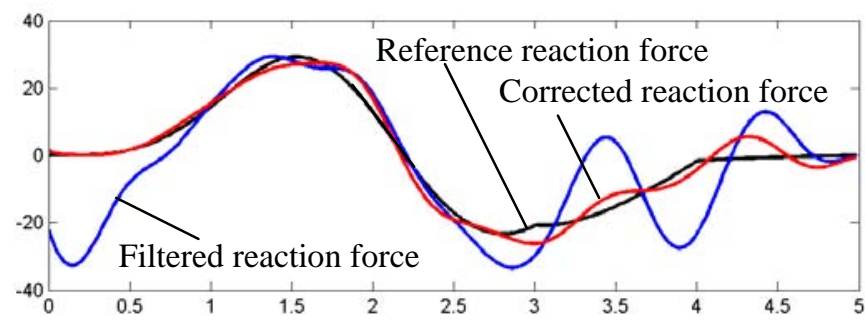

Figure 13: Comparing the reference filtered and corrected reaction force

In the future, we intend to apply this procedure on human motion capture data.

\section{ACKNOWLEDGMENTS}

This work has been supported by the Deutsche Forschungsgemeinschaft (DFG) within the projects M4 of Collaborative Research Center 588

"Humanoid Robots - Learning and Cooperating Multimodal Robots”

\section{REFERENCES}

1. Arnold, M.:

Simulation Algorithms in Vehicle System Dynamics. Reports on Numerical Mathematics

2. Baumgarte, J..

Stabilization of constraints and integrals of motion in dynamical systems.

Computer Methods in Applied Mechanics and Engineering, 1:1-16, 1972.

3. Beth T., Boesnach I., Haimerl M., Moldenhauer J., Bös K., Wank V.

Analyse, Modellierung und Erkennung menschlicher Bewegungen

Human Centered Robotic Systems (HCRS) 2002, 5.6. 12. 2002, Universität Karlsruhe, S. 17-24, 2002.

4. Beth T., Boesnach I., Haimerl M., Moldenhauer J., Bös K., Wank V.

Characteristics in Human Motion - From Acquisition to Analysis

IEEE Int'l. Conference on Humanoid Robots HUMANOIDS 2003, 1.-3. Oktober 2003, Karlsruhe / München, Conference Documentation, S. 56ff, VDI/VDE-GMA, 2003

5. Eich E.,

Convergence results for a coordinate projection method applied to mechanical systems with algebraic constraints.

SIAM J. Numer. Anal., 30:1467-1482, 1993.
6. E. Eich, C. Führer

Numerical methods in Multibody Dynamics

Teubner-Verlag, Stuttgart, 1995.

7. Eich, E., Fuhrer, C., Leihmkuhler, B.J., Reich, S., Stabilization and projection methods for multibody dynamics.

Research Report A281, Helsinki University of Technology, Institute of Mathematics, Finland, 1990.

8. S.K. Ider, F.M.L. Amirouche

Coordinate reduction in the dynamics of constrained multibody systems - A new approach

Journal of Applied Mechanics 55, 1988, 899-904.

9. J. García de Jalón and E. Bayo, Kinematic and Dynamic Simulation of Multibody Systems. The Real-Time Challenge, Springer-Verlag, New-York, 1994, ISBN 0-387-94096-0.,

10. The Mathworks, Inc. Matlab User's Manual, 1996.

11. J. F. O’Brien, R. E. Bodenheimer, G. J. Brostow, J. K. Hodgins

Automatic Joint Parameter Estimation from Magnetic Motion Capture Data

Proc.Graph. Interface, 2000

12. A. A. Shabana

Computational Dynamics

J. Wiley \& Sons, New York, 2001 (2nd Ed.), 520 pp. ISBN 0-471-37144-0

13. M.P.T. Silva, J.A.C. Ambrósio, M. Pereira Biomechanical model with joint resistance for impact simulation

Multibody System Dynamics 1(1), 1997, 65-84.

14. M.P.T. Silva and J.A.C. Ambrosio, Kinematic Data Consistency in the Inverse Dynamic Analysis of Biomechanical Systems,

Multibody System Dynamics 8: 219-239, 2002.

15. R.P. Singh, P.W. Likins,

Singular value decomposition for constrained dynamical systems

Journal of Applied Mechanics 52, 1985, 943-948.

16. Z. Terze, D. Lefeber, O. Muftic

Null Space Integration Method for Constrained Multibody Systems with No Constraint Violation Multibody System Dynamics 6: 229-243, 2001. 